Sustainable Development and Planning X 259

\title{
AIRPORT NEIGHBOURHOODS AS FUTURE REGIONAL DEVELOPMENT AREAS FOR RESOURCE AWARENESS AND GESTALT SUSTAINABILITY: SMARTAIREA
}

\author{
JULIA FORSTER ${ }^{1} \&$ SANELA PANSINGER $^{2}$ \\ ${ }^{1}$ TU WIEN, Austria \\ ${ }^{2}$ Joanneum Research - LIFE, Austria
}

\begin{abstract}
The form of urbanisation has fundamentally changed in recent years. In particular between the airport and the city, and in the airport environment, which is predominantly used by trade and industry, a complex spatial organisation with specific features has developed mostly as a result of logical, economic and political decisions. There is thus a special motivation to transform this spatial complexity in the environment of the airport into a sustainably integrated spatial development for city, region and neighbouring communities - into SmartAIRea. The dynamically growing environment of the airport is an ideal and innovative test bed for intelligent, cross-linked and integrated solutions where sustainability demands can be tried out under multifactorial conditions and implemented as an example throughout Europe, where $40 \%$ of the 460 airports will have to face a potential shut down due to regulatory change. The objective is to integrate the existing airport and its environment not only through ecological, economic and technological synergy and cross-linking, but also by generating an innovative spatial quality which provides gestalt sustainability, thus yielding potential for further urban and regional development. Furthermore the objective is a positioning of a sustainable spatial organization (timeless and time-related), which balances the appearance (gestalt) and its usefulness and in this context sets a framework where people feel alive and present. This process needs concepts which generate and combine smart systems, solutions and methods. The Graz Airport environment thus offers an ideal possibility to embed thematic principles such as building and spatial organisation, sustainable development and energy supply, quality of life, noise reduction, economic balance and good governance in the planning process. The airport environment is transformed into a transparent, interconnected, controllable and resilient location - into SmartAIRea, where the term "gestalt" sustainability, which properly belongs to a people-oriented space, is at centre stage. SmartAIRea as a transferable model can thus give a future-oriented answer to burning questions of sustainable urban development.
\end{abstract}

Keywords: airport area, sustainable urban development, inward development, transdisciplinary participation process, digital modelling and planning support, spatial design, gestalt sustainability.

\section{INTRODUCTION}

The basic condition for the successful accomplishment of today's functional, technological, mobile, energy-optimized, climate-friendly, people-oriented or sustainable requirements in urban and regional development is the space. The spatial organization or the relationship between built-up and non-built space is the essential criterion for smart sustainability aspects. The best inventions and concepts are inappropriate if they do not agree with the space.

Transferred to the airport environment, the impact of the airport location on urban development is conditioned by specific, logical, political and economic decisions of various actors. They aim at better mobility of people and goods and consequent economic growth. But the consequences that they leave behind in the spatial structure are often not considered, not "planned" and thus at least not sustainable. They are reflected in the form of structural aggregates of individually controlled mass accumulation. It is a field that gives the impression of formlessness. 
To illustrate the problem, examples of the airport environment were added - see Fig. 1. It is clearly visible that, from a spatial and traffic planning point of view, something has to happen to integrate this space in urban planning in a sustainable and people-oriented field.

The leaps in scale and discontinuity in the spatial organization, also in the airport environment of the Graz airport, are the results of economic and political developments [4].

\subsection{Motivation}

Especially in this field lies the opportunity to implement a smart innovative technology (industry 4.0, dynamic ridesharing, etc) and to prove its effects (noise, climate, etc.). The Graz airport environment, as a field of clashes of different scales, programs, planning cultures, economic interests and actors, has the chance to become a strong magnet for people, goods and economic activities, taking sustainability into account. The apparent limits in the planning instruments and minds of decision-makers must be overcome in order to connect the region, the city and the communities in a sustainable way (net) and to become an important component of regionally integrated planning and development.

SmartAIRea can contribute to the bundling of future-oriented challenges in the airport environment and helps to accept the new urban planning approaches in terms of sustainability through the simulated and modelled mission statements. An exploratory project will qualify the airport environment.

SmartAIRea is an implementable concept for a high-quality, diverse and comprehensively networked, integrated, sustainable and ultimately smart airport environment. In the foreground, in addition to economic, ecological and social sustainability, is the concept of gestalt sustainability [5], which forms the basis for a people-oriented, high-quality spatial organization.

\subsection{Statement}

SmartAIRea pushes its functions into a field of application that has not been implemented in praxis so far. It is the first common area for the city, surrounding community and their citizens. This problem concerns a lot of hotspots - not only the medium-sized cities in Austria, but also Europe-wide.
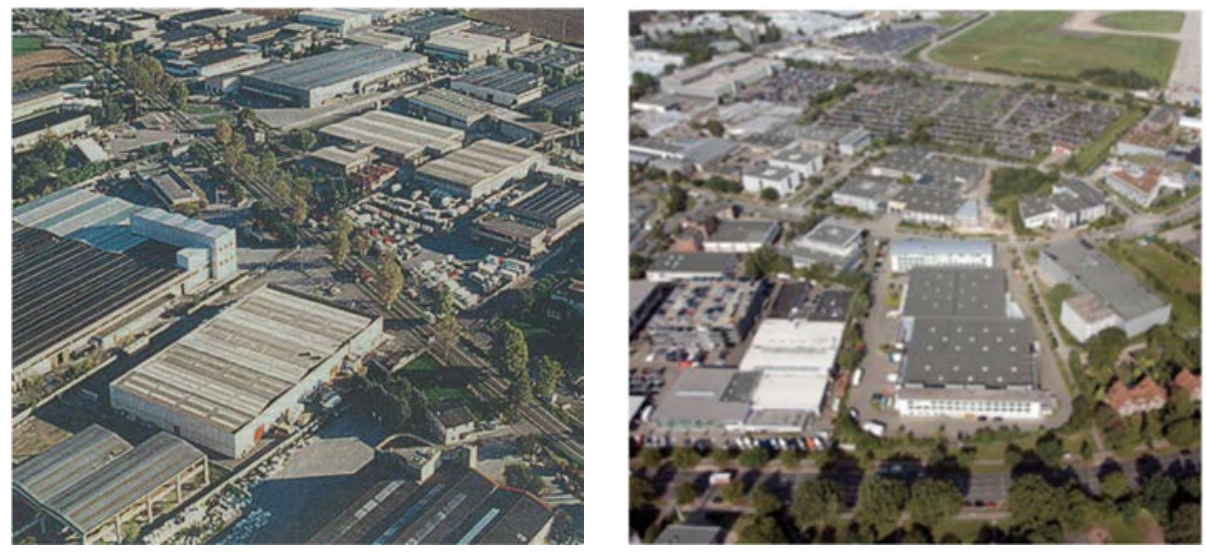

Figure 1: Example of a typical spatial structure between the airport and the city (Verona and Salzburg) [7]. 
The SmartAIRea method is applicable not only to the airport environment, but also in other environments holding controverse poles of interests and functions. Especially if these areas are forming "in between" spatial organisations.

Above all, SmartAIRea shows up-to-date trends of spatial development: station and harbour environment, health complexes, industrial facilities, recreational facilities etc. require after a spatial embedding in their environment, to reach a certain quality of a location. SmartAIRea as a hub of mobile worlds - road, rail, air as an impulse for ecological, spatial and regional development.

\section{STATE OF THE ART}

The reachability effects of global aviation, especially in the environment of hub airports, have so far been addressed by various authors. However, the focus was on the economic or political level. While the effects of airports, such as aircraft noise, are already well analysed in quantitative terms and are always taken into account when planning airports, there are enormous shortcomings in research into the cause-effect relationships of spatial transformation between the airport and the city. Particularly affected are smaller cities - in Austria: Graz.

In addition, it is a relatively new topic of urban development, which has developed only in the late 20th century (such as the low-cost boom since the liberalization of air traffic in 1997) due to complex processes, lacking a temporal distance and the historical analysis, which could explain these processes thoroughly. In doing so, it becomes obvious that today's planning instruments can only insufficiently capture and thus control the structural transformations and their dependence on interactions between the airport location and the city. As a result, there is the risk of further erroneous developments regarding capacities or resources, energy supply, noise pollution, land division or land availability. This makes the consideration of the sustainability aspect particularly difficult.

Air traffic will have to be considered as not sustainable for a long time, because environmentally friendly fuels are still in the development phase. We cannot solve this problem with urban planning, but by integrating smart mobility and energy systems in the airport environment in a sustainable way (simultaneous consideration of the sociological, ecological, economic and design aspects), we can contribute to an improvement. The prerequisite for this is raising awareness of a sustainable airport environment in earlier planning phases. The decisive criterion here is the gestalt sustainability.

The implementation of this sustainable approach in the airport environment offers the possibility to avoid further urban sprawl, to secure landscape, green and open spaces, to promote a suitable mixture of uses in meaningful locations and thus to make a first contribution in Austria or throughout Europe to qualitative urban and regional development. Furthermore, it shows that a relational development of city and airport is possible. Especially the spatially integrated arrangement of the airport environment, as well as spatial implementation of multimodal mobility networks from macro to micro infrastructure space, energy supply systems and structural-technological coupling of climate protection measures motivate the city and the airport operator to reduce conflicts and tensions. The approach allows a mutually transformation of the airport environment instead of separated views of stakeholder interests and infrastructures.

Historically, if port cities were prosperous commercial and economic centres and harbingers of a new, more open society, then airport cities could be the cities of the tolerant future society. 
In the context of solving complex planning problems, one can distinguish between basic components of this system: The planning world, as an outcome of the common sense of a professional planning community, is embedded in the comprehensive world of everyday life.

The life world comprises all kinds of actors, who are involved in or affected by the process of planning and their specific point of view, whereas the planning world works with its own planning approaches, which can vary in different contexts [6, p. $43 \mathrm{ff}]$.

Awareness building processes are trans-disciplinary processes. This means the involvement of actors from planning and life world and requires efforts of time and costs for all participants. For an efficient use of money and time resources within the trans-disciplinary process, Schönwandts 'problems first' approach is a proved proceeding. For fast and effective process realization Schönwandt suggests a concentrated focus on the main challenges within a complex system [2, p. 292]. Regarding a spatial design, process implementation which is integrated within a trans-disciplinary process such as SmartAIRea, means the need for modelling and the use of digital and analogue visualizations. In this regard the visualization depicts a communication base between both, actors of life and planning world [3]. Within planning processes the visualization base allows the definition of actions within space and time which enables the analysis of effects in early planning phases and the comparison of different development paths. Especially interactive visualization environments provide the possibility to interlink arguments of different interests and support within the balancing process of space and time related definitions for strategic development paths. GIS based tools therefor provide useful and easy to handle tools for the development of 3D visualisations for web-based presentations. Via Web Viewers (ex. ESRI CityEngine Web Viewer) or Webmap applications are suitable presentation platforms for fast modelling preparation. The interactive tools present multi-scale analysis for a broad bandwidth of planning participants as well as interested citizens.

\section{CASE STUDY AREA}

Graz is the provincial capital of Styria and with 286.686 inhabitants (as of 1 January 2017) the second largest city in Austria. The Graz surroundings is in the last 10 years the fastest growing conurbation in Austria. The long-term trend shows a steady increase in population of the city of Graz and its surrounding communities, the current forecasts predict an increase in population to around 490,000 by 2050 .

Location of SmartAIRea: The activation field "SmartAIRea" is located south of Graz and bordering the city. The area has a size of about 40ha. The current settlement development in the research area of Graz Airport includes industrial and commercial areas as well as residential areas, mixed areas and many undeveloped areas. Remarkably, water and landfill area next to the airport area is of the same size as the airport area itself - Fig. 2.

Thus there is a risk that the Graz airport environment, as an important mobility hub, will be characterized by urbanized, faceless and non-organized suburban structures that are neither urban, architectural nor sustainable. Together with the airport operating area, it then creates a spatial obstacle and generates further urban sprawl in the Graz periphery.

Because of the airport noise and the proximity to the airport, train and highway is the apparent reason of the previous regional and urban political intentions that the airport environment is only suitable for the economically oriented industrial and commercial sites. The absurdity is that on the other, western side of the airport, even adjacent to it, the allotments have settled on monopolized man-made waterways and south-east of the airport residential family housing estates, providing proven advocacy for the day-to-day, recreational and leisure activities in the airport area can be found. This fact or the resulting improbability (investor architecture, property speculation, re-allocations, etc.) in the spatial 


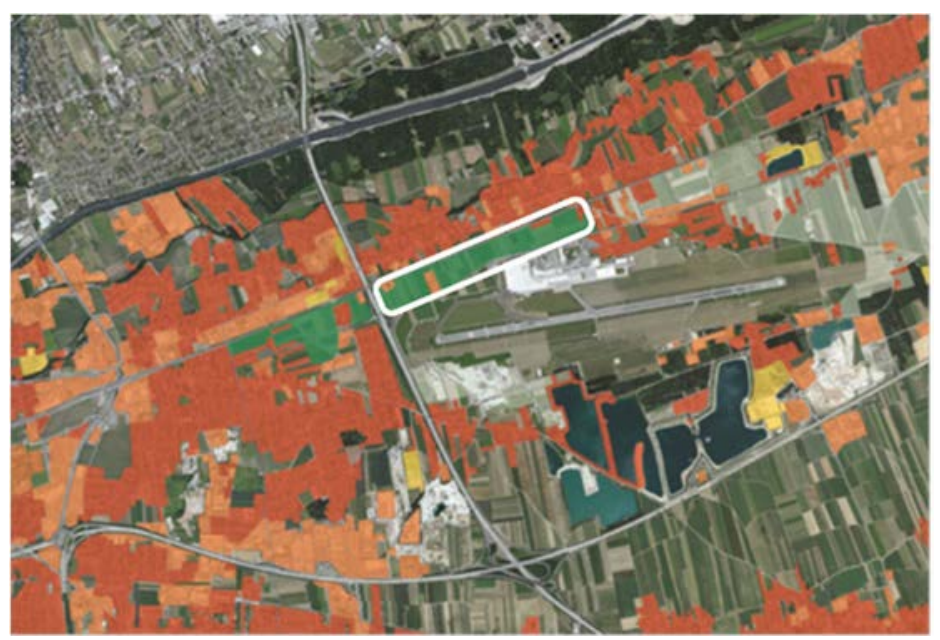

Figure 2: Field of view of the SmartAIRea is shown in green colour and white circles. Colour Red $=$ Living, Colour Orange $=$ Trade and Technology. Verona - Otopiu Casa. Giornale di Brescia; Salzburg [8].

organization indicates that the airport environment is often a place where conflicts of interest have so far only been insufficiently resolved.

\section{4 "GESTALT SUSTAINABILITY" AS METHODOLOGY WITHIN TRANS-DISCIPLINARY PLANNING PROCESSES}

The fact that all our actions are deposited in space and take shape without always being the result of a design process, provides the possibility of a conscious decision to accompany this deposition process as a design process, to give the figure a sustainable added value and thus to make an impact on society, the environment and the economy.

Sustainable projects and processes will only be successful if social, ecological, economic and design as "Gestalt" aspects become a matter of course.

By consideration the gestalt sustainability, which was defined and applied for the first time by the project SmartAIRea (www.smartairea.eu), activates the space in the long term and thus conserves resources, saves energy, is climate-friendly and ultimately efficient.

Thus, the goal of gestalt sustainability is the preservation, in terms of scale and time, of the essential identity-forming properties of the spatial structures.

As a result, the following key concepts for gestalt sustainability in the airport environment can be derived

- Location - character / genius loci

- Purpose/monofunctionality - multifunctionality

- Sense of scale

- Infrastructural embedding

- Organizational dimension/long and short life

These key-terms of gestalt sustainability, in terms of resource and energy-related aspects in the airport environment, will allow the airport field to position itself as a networked, responsive, transparent and controllable location. 
Since it is always about the systematic units and their spatial implementation and consideration as a whole, the key concepts in formulating the gestalt sustainability aim at the following focal points:

\subsection{Smart industrial site/commercial area}

- The industrial building should be characterized by its optimal spatial position and infrastructural connection to achieve a reduction of emissions.

- The design considerations, taking into account the spatial and building organization of the industrial buildings, place special emphasis on repair and maintenance properties, the value retention as well as the improvement of recyclability and suitability for conversion.

- The sustainable construction, spatial integration through the green and public space and the use of intelligent systems creates a liveable area that makes a significant contribution to a sustainable future.

- The existing and future buildings should be intelligently networked with each other.

\subsection{Smart energy}

- In the field of energy consumption, a reduction of emissions via new sustainable developments concerning spatial organization should be achieved. On the one hand energy-efficient heating technology and air conditioning, hot water preparation, device replacement, lighting concepts and on the other by intelligent networking of the actors are essential drivers for successful sustainability transition.

- The aim is to ensure self-sufficiency, largely based on alternative energy sources. The energy gained should be available for the building's own needs, goods transport and electric mobility. Surpluses should be stored and thus used again for the building network.

- In addition to power generation technologies, energy exchange also includes the buildings between which energy (electricity, heat) is exchanged, as well as the grid infrastructures necessary for energy transport.

- The smart energy strategy is inseparable from spatial and building organization, linked to infrastructural embedding and multi-optional mobility.

\subsection{Smart mobility}

- The topic of working, living or everyday activities also aims to reduce $\mathrm{CO}_{2}$ emissions. This aim is to be achieved by using sharing solutions and creating multimodal transport hubs, that have the character of public space and enable the transition to green mobility.

- Multimodality should be integrated into the existing traffic structure (train, motorway, airport) in a user-friendly way.

- Concerning passenger mobility, highly visible E-sharing hot spots will be built to achieve $\mathrm{CO}_{2}$ reduction.

- The smart mobility concept such as autonomous driving should also be linked to the architecture and the space and adapted to the space conditions in order to be efficient. 


\subsection{Smart environment}

- On the one hand, the development of an innovative transport and environment concept has to take into account the environment and the creation of green spaces and traffic-calming zones. On the other hand, it involves the inclusion of the environment within the framework of design sustainability.

\subsection{Gestalt sustainability means handling of proportions and needs 3D thinking}

3D visualisation enables the imagination of scale and perspective for involved stakeholders in planning processes. People perceive gestalt as a sum of form, proportion and material.

On an architectural scale, this means the gestalt of facades or room configurations. On scale 1:500 and upwards it means the proportion between developed and undeveloped areas. Moreover it means the kind of development and applied materials. Sealed vs. unsealed ground and built height are the main keys for the individual perception of spaces. Thus 3D modelling is an essential tool for the design as well as the conveyance of ideas.

\subsection{Analog and digital models as visual communication bases and strategic planning environments}

Tackling inter and transdisciplinary challenges within urban development regions means the identification of problems within spatial context and time. For the development of resource efficient strategies, the definition of actions as well as the assessment of consequential effects must be covered within cross-domain planning processes. An efficient strategy process covers a round table meeting involving actors from the planning and life world. For presentation purposes as well as communication base, spatial models are planning and decision supply tools. To spatially pinpoint planning challenges the design of spatial models depicts essential and vital features for the design of planning related ideas and actions $[1$, p. 27]. Thus a digital simulation environment was developed which holds the built building stock and illustrates the area related usages. A digital image of reality enables the visualisation of new building developments and alternatives. Thus obtained findings and results can be analysed in multi scale levels. Furthermore quantitative information can become attached to single objects within the model. Calculation outputs from various disciplines are connectable and holistic system views are enabled. Thus the digital environment allows the visualisation of energy and mobility related data as examples. The connection of geometrical and alpha-numerical model objects enable digital storage of analogue data. In this way, data and findings can not only be stored long term but also passed on to others and used elsewhere.

For the inter- and transdisciplinary planning process a detailed model of the SmartAIRea was designed. The model covers the existing buildings within the topological formations. Based on this model a haptic model was milled and laser-cutted - Fig. 3 shows the elaborated model and its usage within a strategy development workshop.

To illustrate outputs and progress findings, alternatives were implemented within the digital model. Thereby detailed measures as well as abstract ideas are spatially pinpointed side by side.

For the communication of main project outputs and to raise awareness for potentials within the project area to a broad bandwidth of people an interactive visualization environment was set up. The interactive environment is based on a web-based $3 \mathrm{D}$ viewer technology (City Engine Web Viewer). Fig. 4 shows a screenshot of viewer and model. 
Within the viewer two different alternatives as well as main potentials within the region are spatially visualised. The web-based technology allows the usage for a broad bandwidth of people.

\section{RESULTS}

The results of SmartAIRea presented in Figs 5 and 6 are based on scenarios and became visualized in 3D environments for communication purposes under www.smartairea.eu.

Fig. 5 shows scenario 1, which suggests the development of a climate park (left). Furthermore, it allows comparing this scenario with scenario 2, which presents short paths via highly efficient mix of use (right). For both scenarios, a holistic mobility concept is essential. Fig. 6 illustrates a possible development path for e-mobility implementation (blue) as well as the future railroad "Koralmbahn" is highlighted (orange).

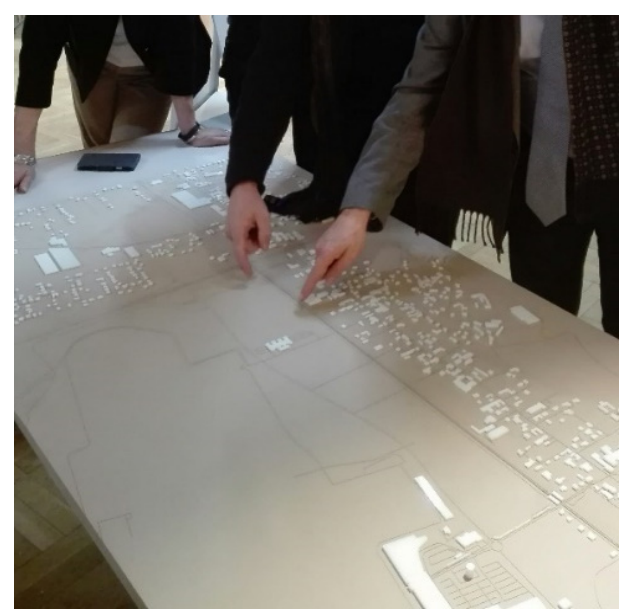

Figure 3: Static (analog) model design [9].

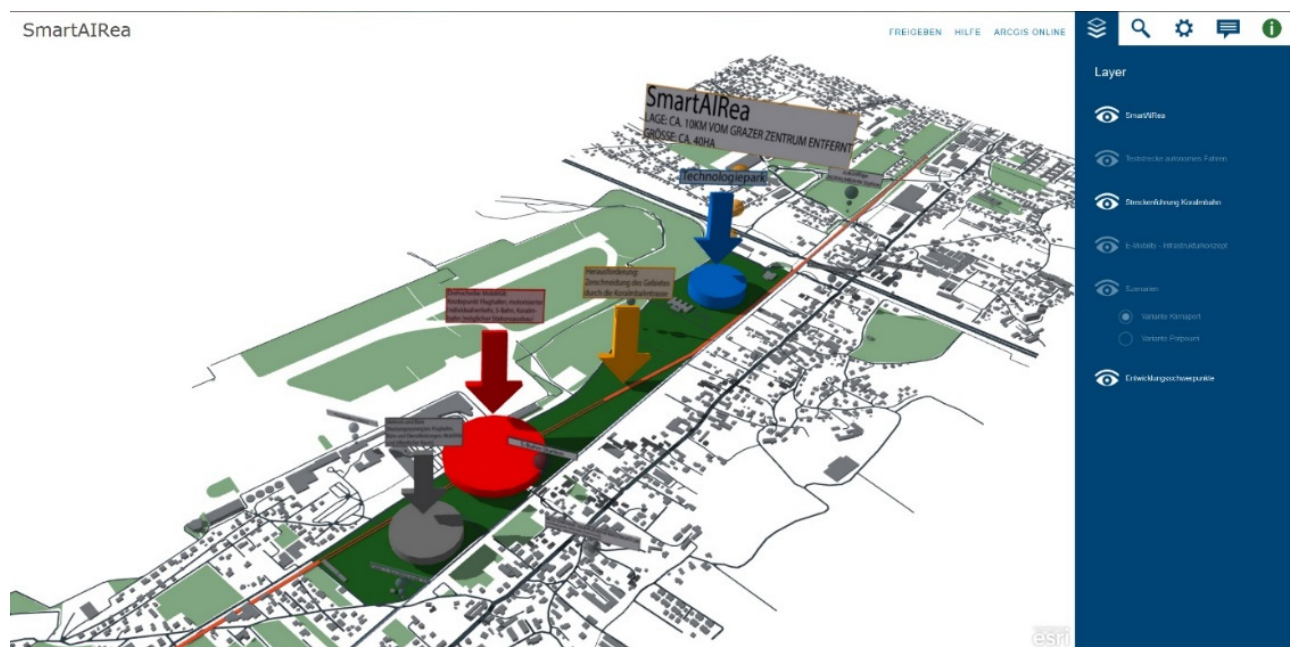

Figure 4: Parametric (digital) model design [10]. 

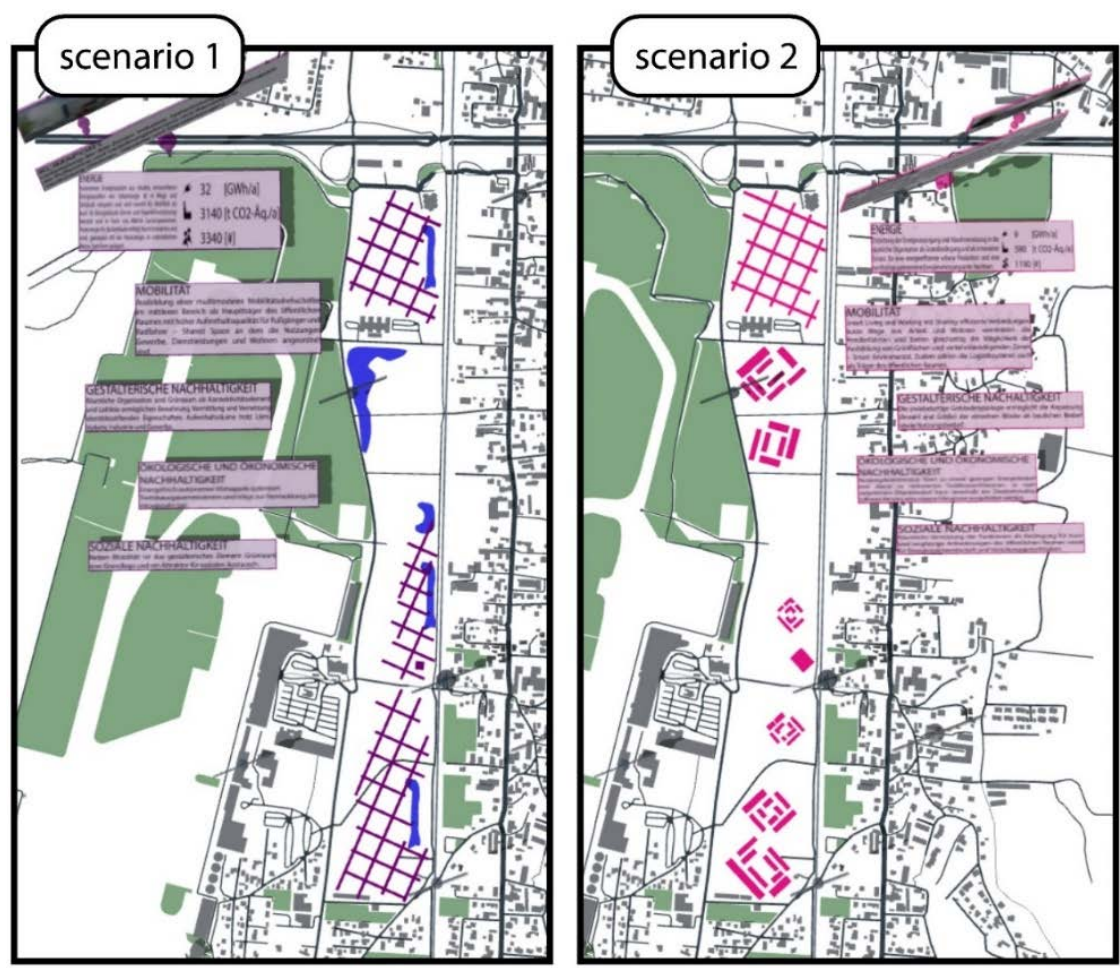

Figure 5: Comparison of scenario [10].

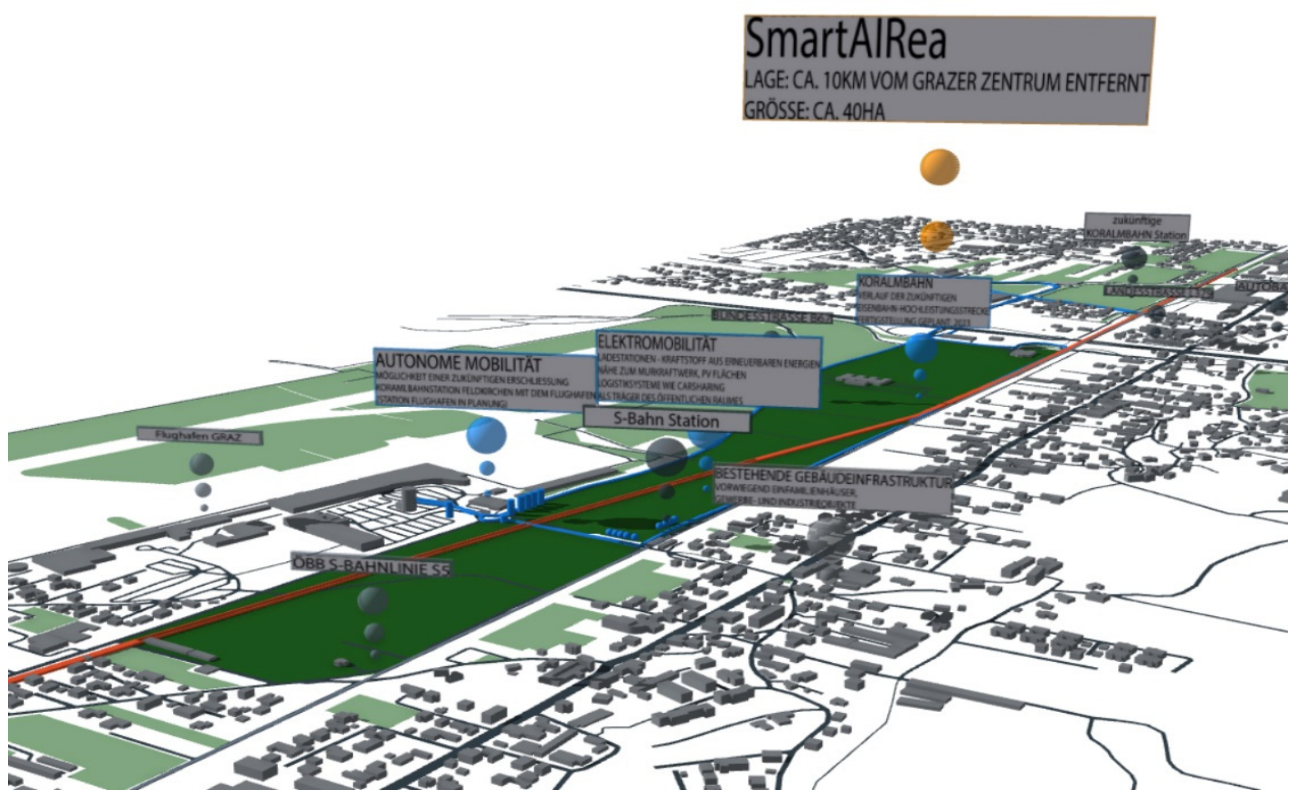

Figure 6: Screenshot of homepage www.smartairea.eu [10]. 


\section{FINAL REMARKS}

SmartAIRea has an innovative task of great importance:

To transform the condensed and controversial claims for the first time into a balanced and sustainable space organization of the airport environment.

Decisive for the success and associated risks of SmartAIRea concept are easy-tounderstand initiatives and recommendations that are tailored to the needs of the players in the airport environment as well as the opportunities and interests of involved research partners.

SmartAIRea will thus provide insights into the player-specific attitudes and behaviour in the airport environment of medium-sized cities as well as a new basis for strategic orientation of cities with airports in terms of energy and climate awareness.

SmartAIRea represents a present method of dealing with the different levels of planning concerning society, economy, planning and politics. The critical reflection of development directions of cities and surrounding communities, based on cooperation and communication, is the primary prerequisite for energy and climate-conscious regional and urban planning.

SmartAirea - a transferable concept, a space organization with a memory value and its own Genius Loci, where the term gestalt sustainability is first defined and used.

\section{REFERENCES}

[1] Voigt, A., Raumbezogene Simulation und Örtliche Raumplanung, Österreichischer Kunst und Kulturverlag, Wien, 2005.

[2] Schönwandt, W., Methoden der Raumplanung Akademie für Raumforschung und Landesplanung (Hrsg.). Grundriss der Raumordnung und Raumentwicklung, Hannover, 2011.

[3] Forster, J., Strategische raumbezogene Visualisierung im Kontext der Innenentwicklung urbaner Siedlungs-, Energie- und Mobilitätssysteme am Beispiel der Stadt Wien, TU Wien, Dissertation, 2016.

[4] Pansinger, S., Flughafenstandort - Stadtentwicklung. Das Dazwischen als Abstellraum, TU Graz, 2015.

[5] Pansinger, S., Gestalterische Nachhaltigkeit - Handlungsfeld der Zukunft, unseren ökologischen Fußabdruck zu verkleinern, Smart Cities, Wien, 2016.

[6] Grunau, J.-P., Lösen Komplexer Probleme. Theoretische Grundlagen und deren Umsetzung für Lehre und Praxis, Tönning, Lübeck, Marburg: Der Andere Verlag, 2008.

[7] Google Maps.

[8] Orthographic aerial photograph, BEV - Bundesamt für Eich- und Vermessungswesen, Vienna as editing document, graphic: Pansinger.

[9] Picture by Forster 2017.

[10] Illustration by Forster and Pansinger 2017. Online. www.smartairea.eu. 